\title{
PERFORMANCE OF THE AFS VERTEX DETECTOR AT THE CERN ISR
}

\author{
Brookhaven-CERN-Copenhagen-Lund-Rutherford-Tel Aviv Collaboration
}

O. BOTNER, V. BURKERT, D. COCKERILL, C.W. FABJAN, T. FERBEL, P. FRANDSEN, A. HALLGREN, B. HECK, H.J. HILKE, R. HOGUE, P. JEFFREYS, H.B. JENSEN, T. KILLIAN, M. KREISLER, J. LINDSAY, H. LUBATTI, T. LUDLAM, W. MOLZON, B.S. NIELSEN, Y. OREN, P. QUERU, L. ROSSELET, E. ROSSO, A. RUDGE, R. SCHINDLER, M. SCIRE, D.W. WANG, Ch.J. WANG, W.J. WILLIS, M. WINIK

CERN, European Organization for Nuclear Research, Geneva, Switzerland

H. BØGGILD, E. DAHL-JENSEN, I. DAHL-JENSEN, Ph. DAM, G. DAMGAARD, K.H. HANSEN, J. HOOPER, R. MOLLER, S.Ø. NIELSEN, L. OLSSEN, B. SCHISTAD

Niels Bohr Institute, Copenhagen, Denmark

T. AKESSON, S. ALMEHED, G. von DARDEL, S. HENNING, G. JARLSKOG, B. LÖRSTAD, A. MELIN, U. MJÖRNMARK, A. NILSSON

University of Lund, Lund, Sweden

M.G. ALBROW, N.A. McCUBBIN, W.M. EVANS

Rutherford Laboratory, Chilton, Didcot, England

O. BENARY, S. DAGAN and D. LISSAUER

Tel Aviv University, Tel Aviv, Israel

The central detector of the axial field spectrometer (AFS) is a cylindrical drift chamber using a "bicycle-wheel" geometry. Its design has been optimized for jet-like events with high track densities. This is accomplished through a high degree of azimuthal segmentation ( $4^{\circ}$ sectors) with up to 42 space points per track, using measurements of drift time and charge division. Particle identification in the non-relativistic region is obtained by $(\mathrm{d} E / \mathrm{d} x)$ sampling. The detector is operated in an inhomogeneous magnetic field at event rates of typically $5 \times 10^{5}$ collisions per second. Results will be presented on the detector performance after one year of operation at the ISR.

\section{Introduction}

The axial field spectrometer (AFS) at the CERN ISR was designed to study events characterized by large transverse momenta. It required a low mass vertex detector providing good momentum resolution in a solenoidal magnetic field of $0.5 \mathrm{~T}$, powerful pattern recognition for high event rates and particle densities and particle identification in the non-relativistic region [1].

0029-554X/82/0000-0000/\$02.75 (c) 1982 North-Holland

\section{Chamber design and operation}

A cylindrical drift chamber with $4^{\circ}$ azimuthal segmentation was constructed [2], combining drift time measurement for the azimuthal coordinate with charge division read-out for the coordinate along the wires (fig. 1). The chamber is $1.4 \mathrm{~m}$ long and extends from a radius of $0.2 \mathrm{~m}$ to $0.8 \mathrm{~m}$. Each $4^{\circ}$ sector contains 42 resistive sense wires ( $30 \mu \mathrm{m}$ diameter, $8 \mathrm{~mm}$ spacing), arranged radially into three crowns. The sense wires are 

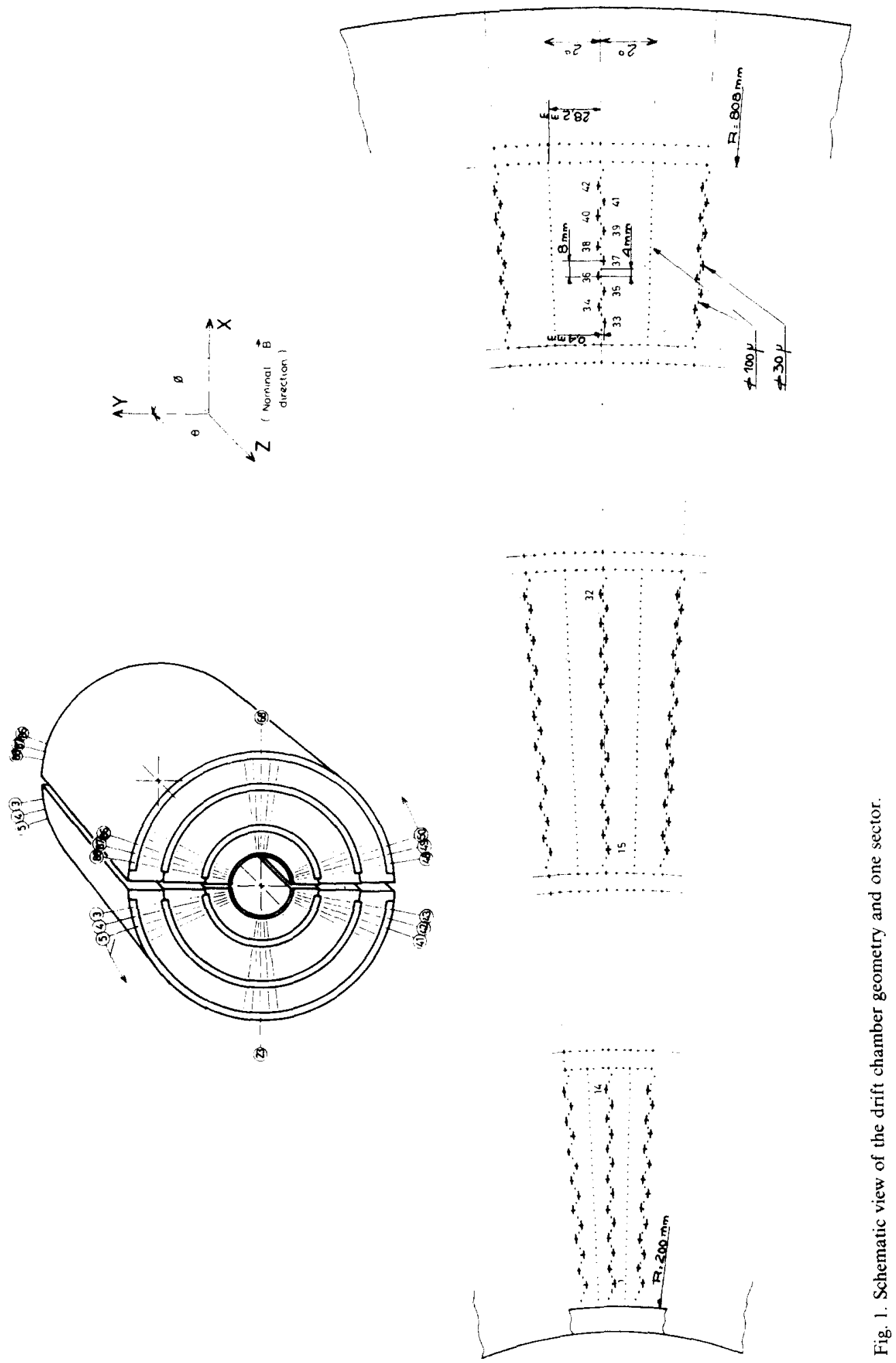
staggered by $\pm 0.4 \mathrm{~mm}$ to resolve the left-right ambiguity. Sector boundaries consist of grids of $100 \mu \mathrm{m}$ wires. Particle identification in the non-relativistic region is obtained from multiple ionization sampling $(\mathrm{d} E / \mathrm{d} x)$. The drift chamber was built in two half-cylinders to permit rapid retraction from the magnet. The outer section of the chamber experiences magnetic field inhomogeneities of more than $30 \%$, due to the open, magnet structure.

The chamber is operated with an argon/ethane mixture $(50 / 50$ vol.\%) at atmospheric pressure. A gas amplification of $(3-6) \times 10^{4}$ is chosen as compromise between acceptable chamber lifetime and $\mathrm{d} E / \mathrm{d} x$ resolution on the one side and good space resolution on the other.

\section{Performance}

\subsection{Drift time measurements}

The CERN DTR units are used for drift time digitization, contributing $\sigma=60 \mu \mathrm{m}$ to the space resolution. For all tracks, visible radially over $>50 \mathrm{~cm}$, we obtain a point resolution with fwhm $/ 2.35=200 \mu \mathrm{m}$, averaged over the full azimuth and all points with valid $z$ information. The residue distribution which is well approximated by a Gaussian in the central part, contains, however, stronger tails. Near the sense wires and the sector boundaries the resolution is worse, in between these zones it is better.

Corrections are applied for electrostatic wire deflections, magnetic field inhomogeneities, drift velocity variations and also for the time slewing and crosstalk ( -4 to $-6 \%$ ). A substantial improvement was gained by the introduction of the last two corrections: a general time slewing term, similar for all wires, and an additional crosstalk term only for the "far wires". These wires, because of staggering, receive their avalanche signal only some $16 \mathrm{~ns}$ after the crosstalk signal from the adjacent wires and, therefore, experience additional time slewing.

\subsection{Charge division}

As expected, a strong correlation is observed between the resolution from charge division and pulse height $(\mathrm{PH})$ : fwhm $(\mathrm{cm})=A+B$.noise $/ \mathrm{PH}$. For a gas amplification of $5 \times 10^{4}$, the average resolution for all reconstructed tracks is $\mathrm{fwhm} / 2.35=1.4 \mathrm{~cm}$, i.e. $1 \%$ of the wire length.

\subsection{Particle identification by $d E / d x$}

Averaging over all tracks containing $>30$ time digitizations and $z$-measurements, we obtain for the trun-

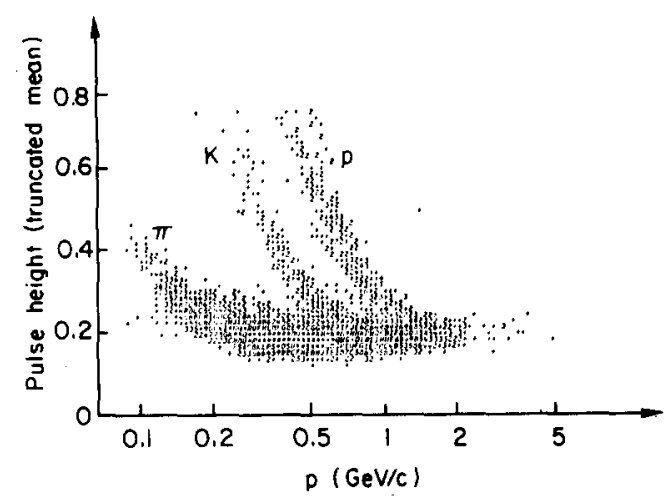

Fig. 2. $\mathrm{d} E / \mathrm{d} x$ : truncted mean vs. momentum. Clear $\pi / \mathrm{k} / \mathrm{p}$ separation is achieved in the non-relativistic region.

cated mean of the $60 \%$ lowest pulse heights: fwhm $/$ mean $=24 \%$, a value very close to that predicted by Monte Carlo estimation. As fig. 2 shows, protons may be identified up to $1.0 \mathrm{GeV} / c$, pions and kaons up to $0.6 \mathrm{GeV} / c$. For high particle densities, the superior granularity of this system offers a substantial advantage over a standard time-of-flight system. As an illustration of the performance in complex events, the preliminary spectrum of the total charged tranverse energy $E_{\mathrm{T}}$ is shown in fig. 3, for $\mathrm{pp}$ and $\alpha \alpha$ collisions. It was obtained

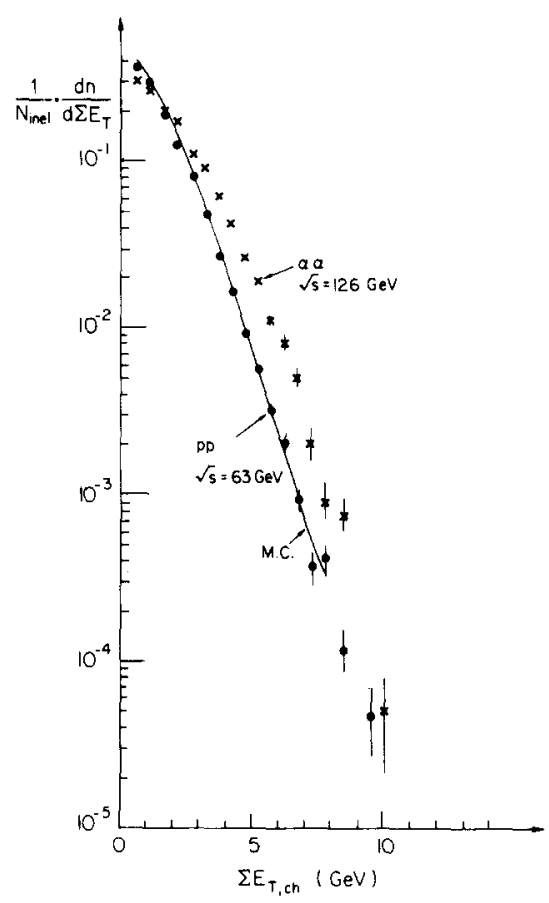

Fig. 3. Total charged transverse energy $\left(E_{\mathrm{T}}\right)$ spectrum, obtained from momentum measurement and particle identification by $\mathrm{d} E / \mathrm{d} x$ in the drift chamber. Curve from Monte Carlo fit. based on inclusive spectra and multiplicity distributions. 


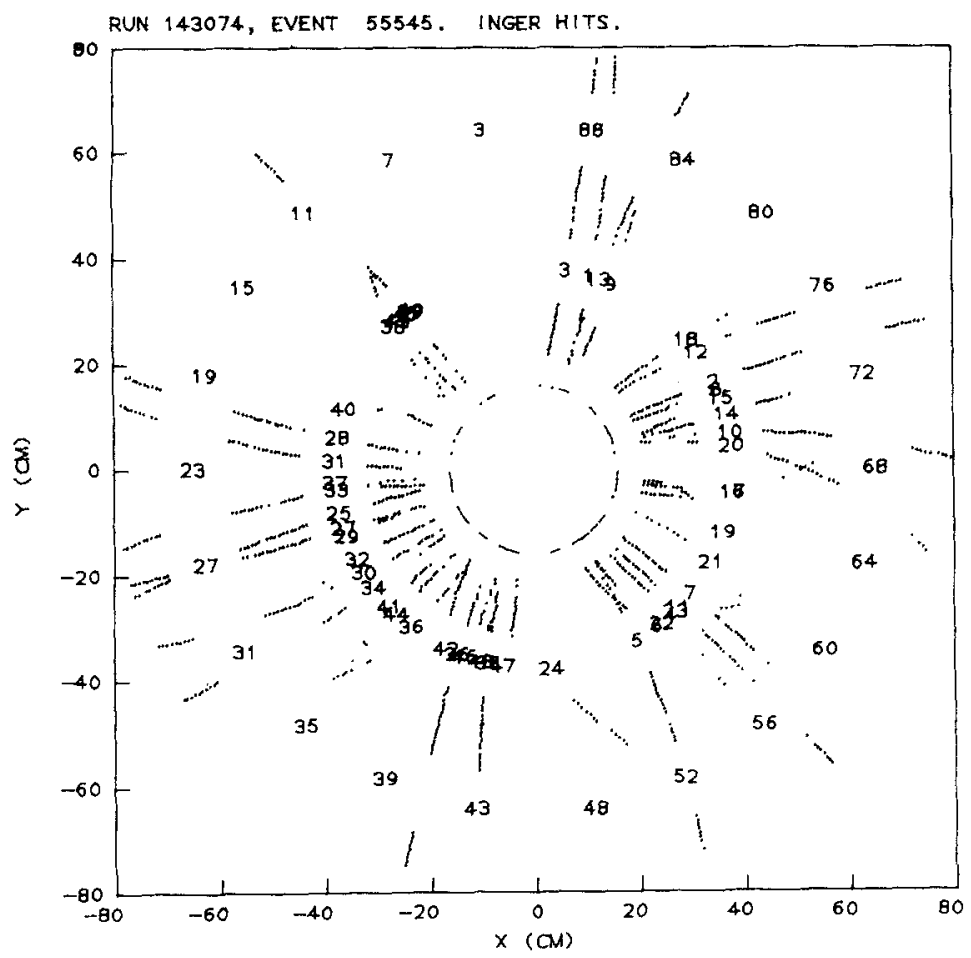

Fig. 4. High multiplicity event with 45 tracks reconstructed. Minimal calorimeter trigger on an $\alpha \alpha$ interraction at $\sqrt{s}=126 \mathrm{GeV}$.

with minimum bias trigger and uses only particle identification in the drift chamber, assuming the pion mass, whenever no identification is obtained. A simple Monte Carlo fit is added, based on measured multiplicity distributions and inclusive spectra, which well reproduce data from other experiments.

\subsection{Diverse}

The track reconstruction efficiency of the analysis program is greater than $95 \%$, even at high multiplicities $\left(N_{\text {ch }}>20\right)$. An example of an event containing 45 reconstructed tracks from an $\alpha \alpha$ collision is shown in fig. 4. Data from cosmic rays are being actively studied for surveying purposes and for obtaining better knowledge of systematic errors, e.g. for momentum measurements.

In parallel, a laser beam was set up to produce ionization tracks in one sector of the drift chamber, with magnet on and off conditions. The preliminary results are very encouraging. Clean tracks were observed all through the sector, although the beam had to cross field shaping wires. A fit to $40 \mathrm{~cm}$ of the track (28 wires) gave $\sigma_{r \phi}=100 \mu \mathrm{m}$ (from drift time) and $\sigma_{z}<1 \mathrm{~cm}$ (from charge division). This includes $\sigma_{r \phi}=60 \mu \mathrm{m}$ from the electronics and indicates that the wire positions and electronic delays are well known. The inner track segment was excluded from the fit, as the laser beam crossed the sense wire plane and produced disturbing pulses on the potential wires. The pulse height distribution showed a fwhm $/$ mean $<25 \%$ for single wires, including fluctuations in the laser intensity.

The advantages of the laser beam for calibration and monitoring are: no magnetic deflection, no production of $\delta$ electrons, narrow amplitude spectrum and precise and reproducible localization in space and time [3]. Some calibration errors - not recognized before - become obvious from the first few events. From a detailed study of these measurements we expect a substantial improvement of the detector performance through better knowledge of various parameters as drift velocity and drift angle variations, time slewing and other systematic effects.

\section{References}

[1] S. Almehed et al., CERN/ISRC/76-36/P95 (1977);

H. Gordon et al., these Proceedings, p. 303.

[2] D. Cockerill et al., Nucl. Instr. and Meth. 176 (1980) 159; J.C. Berset et al., Nucl. Instr. and Meth. 176 (1980) 251;

D. Cockerill et al., Physica Scripta 23 (1981) 649.

[3] H.J. Hilke, Nucl. Instr. and Meth. 174 (1980) 145. 